\title{
Additive Manufacturing for Enhanced Cooling in Moulds for Casting
}

\author{
Even Wilberg Hovig, Vegard Brøtan, Knut Sørby \\ Department of Production and Quality Engineering \\ Norwegian University of Science and Technology \\ Trondheim, Norway
}

\begin{abstract}
Additive manufacturing allows new design solutions for moulds and dies that can improve quality and productivity in casting processes. Complex cooling channels made by additive manufacturing give fast and reliable cooling and a more accurate control of the solidification. The paper shows a comparison of the cooling time of a die insert with a conventional cooling system and an insert with improved cooling channels. It is shown that improved cooling channels are highly efficient even with air cooling. Air could replace water as cooling medium, which will eliminate the explosion hazard in aluminium casting.
\end{abstract}

Keywords-additive manufacturing; low-pressure die casting; conformal cooling

\section{INTRODUCTION}

Additive manufacturing has developed to be a technology suitable for the manufacturing of high-quality tools in the aluminium casting industry [1]. This enables the toolmaker to produce more advanced tools that further enhances the control of the casting process. Improved tooling and process control can improve the casting quality and increase the productivity of the process.

Thermal control is imperative to a good end-result in lowpressure die casting. Additive manufacturing enables the use of cooling channels that follow the shape of the mould cavity and give fast and uniform cooling, usually called conformal cooling channels. The effect of conformal cooling channels will be investigated in this article.

\section{ADDITIVE MANUFACTURING AND LOW-PRESSURE CASTING}

\section{A. Additive manufacturing processes}

Additive manufacturing processes allows rapid manufacturing of physical objects based on three-dimensional CAD-files. A variety of materials can be used in such processes, including polymers, ceramics, and metal alloys. One of the technologies for additive manufacturing of metal alloys is the technology selective laser melting (SLM).

In the SLM-process a laser fully fuses a metallic powder layer by layer until the desired geometry is created. The process consists of three main steps: First, a roller or scraper distributes a layer of fine metallic powder on a build plate. Secondly, a laser traces the shape of the object to be manufactured to fuse the powder into solid material. Lastly, the building platform is lowered, and the powder distribution chamber is raised. The process then repeats itself until the object is finished.

After an SLM-process is complete a sequence of finishing operations is necessary to meet customer demands. These operations include, but are not limited to, removal of excess powder, cutting the object from the build plate, heat treatment, conventional machining, and/or surface finishing.

In additive manufacturing, complexity does not drive the cost. It is therefore a suitable process for making complex parts, especially parts with complex internal shapes. One benefit of using additive manufacturing in casting dies is the possibility to create conformal cooling channels that follows the geometry of the cast part [2]. The technology is already used in the making of tools in the plastic injection moulding industry $[3,4]$, but the use of additive manufacturing in the making of dies for low pressure die casting is still limited.

\section{B. Low-pressure die casting}

Low-pressure die casting is used to produce parts with high integrity and in large volume, typical examples are aluminium castings for the automotive industry. Sand cores or metal cores can be incorporated in the process to produce hollow parts.

In low-pressure die casting the molten metal is forced into the mould through a fill tube that is immersed in a crucible below the mould. The air around the crucible is pressurized to generate the necessary force to lift the molten metal into the mould. A schematic illustration of a low-pressure die casting

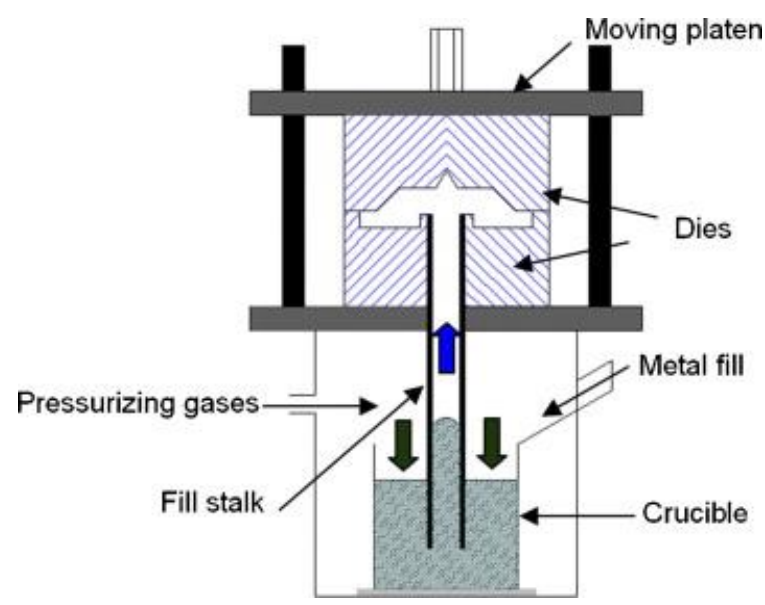

Fig. 1. Low-pressure die casting machine [5] 


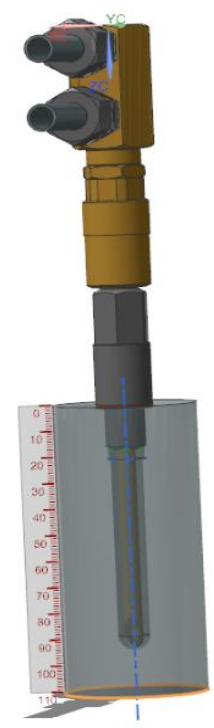

Fig. 2. Conventional cooler used in die casting

machine is shown in Fig. 1. A distinct advantage of the process is that clean molten metal is introduced into the fill tube, and surface oxides from the melt are avoided in the mould.

Generally, post processing costs are low because there are no raisers that must be removed from the casting, and there is often only one inlet point that must be trimmed. Post processing of components can include removal of sand cores, removal of flash, and heat treatment.

As the metal fills the mould, the air inside the mould must be evacuated through properly located venting elements, or through narrow gaps between mould inserts. If air, or gas from the sand cores, is trapped inside the mould the result is incomplete filling of the mould and imperfect castings. The defects could be found on the surface of the casting, or inside the casting. Ventilation is often combined with ejector pins. If larger venting area is desired, inserts with venting slots can be incorporated in the mould.

The steel mould has a large mass compared to the casting, and the molten metal could solidify quickly in contact with the
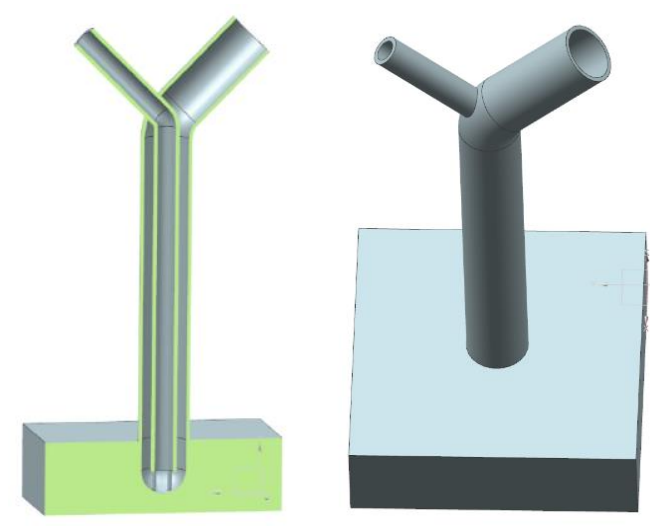

Fig. 3. CAD model of point cooler used in the experimental study wall of the mould. On one hand it is advantageous to cool the molten metal fast to reduce the cycle time, but on the other hand too fast cooling could lead to imperfections in the casting. A controlled solidification process is necessary to achieve good mould filling and desired microstructure properties. The cooling process is usually controlled by water cooling. Electrical heating elements in the mould can also be used for thermal control.

\section{Moulds and dies based on additive manufacturing}

Cooling channels in dies are usually made by conventional metalworking processes, which means that the channels have a simple geometry. The cooling channels are relatively ineffective and requires large coolant flow to achieve the desired cooling effect. An example of a conventional cooler is shown in Fig. 2. The cooler has an outer tube and an inner tube which separates the ingoing and outgoing flow. The cooler will reduce the temperature in a small area of the die, and therefore several coolers and a high number of hose connectors could be needed. The increased complexity of the mould and the casting machine will reduce the robustness and reliability of the process.

To improve the cooling channel geometries, machined dies with straight cooling channels can be replaced by dies made by additive manufacturing. Conformal cooling has become a wellestablished principle when producing moulds in additive manufacturing with cooling channel closely following the surface profile of the cavity. The use of conformal cooling channels is already seen in industries such as plastic injection moulding, blow moulding, sheet metal forming, and to some extent bulk forming such as extrusion, rolling, and forging [3, 6]. Conformal cooling enhances thermal control and can potentially drastically lower cycle times. A larger cooling area can easily be achieved and the channels could be designed to have relatively low flow resistance. Externally the die would have only a small number of connectors which would simplify setup and maintenance of the casting machine.

In low-pressure die casting conformal cooling can be applied to areas of the mould where thermal control is challenging, or in larger areas to reduce the cycle time of the process.

In addition to advanced cooling channels, additive manufacturing can be used to produce die inserts with venting slots to evacuate air and core gas. Such venting systems has successfully been demonstrated both in injection moulding of

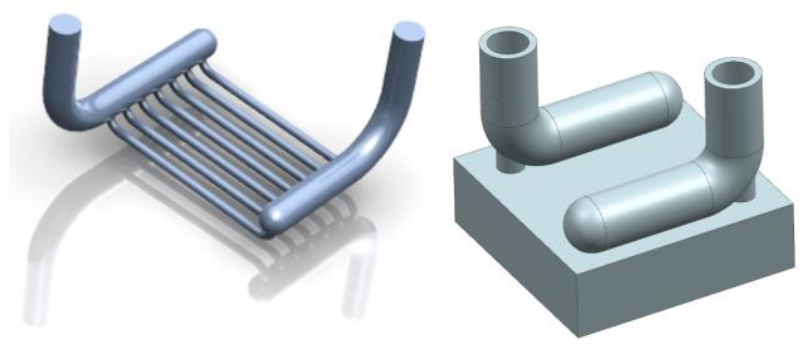

Fig. 4. CAD model of conformal cooler used in the experimental study 


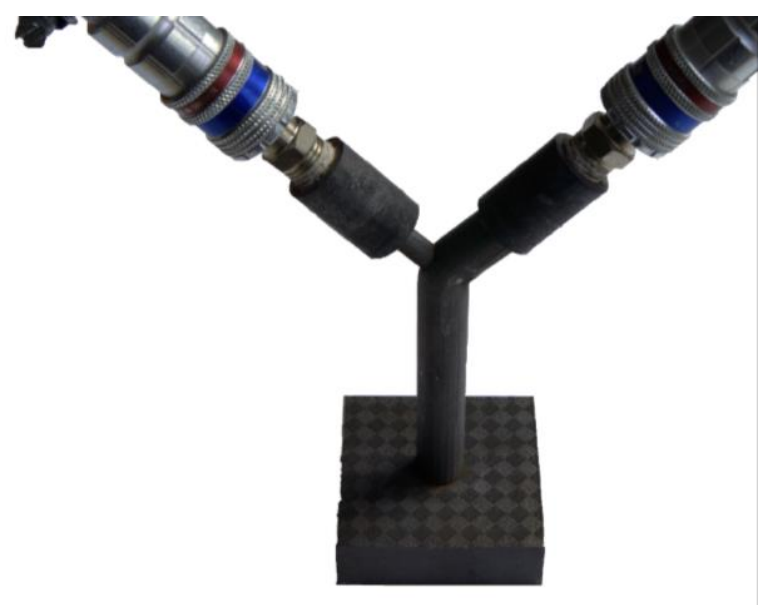

Fig. 5. The physical test objects for point cooling.

polymers and in low-pressure die casting of aluminium. The results presented shows that dies and moulds made by additive manufacturing can improve the part quality, increase the productivity and also extend the life of the tooling $[4,6]$.

\section{EXPERIMENTAL STUDY}

\section{A. Test objects and test procedure}

To quantify the improvement of the cooling effect achieved by complex cooling channels in an additively manufactured die, a test was carried out to compare the new design with conventional cooling channel geometry. The cooling channels were incorporated in a prismatic steel object of the size $60 \times 60 \times 18 \mathrm{~mm}$. To keep factors like heat capacity and thermal conductivity constant in the tests, both test objects were made from the same material in an additive manufacturing process.

The geometry of the test object for conventional cooling the point cooler - is shown in Fig. 3. The inlet water is connected to the outer tube. The improved cooling channel geometry that utilizes the advantages of additive manufacturing

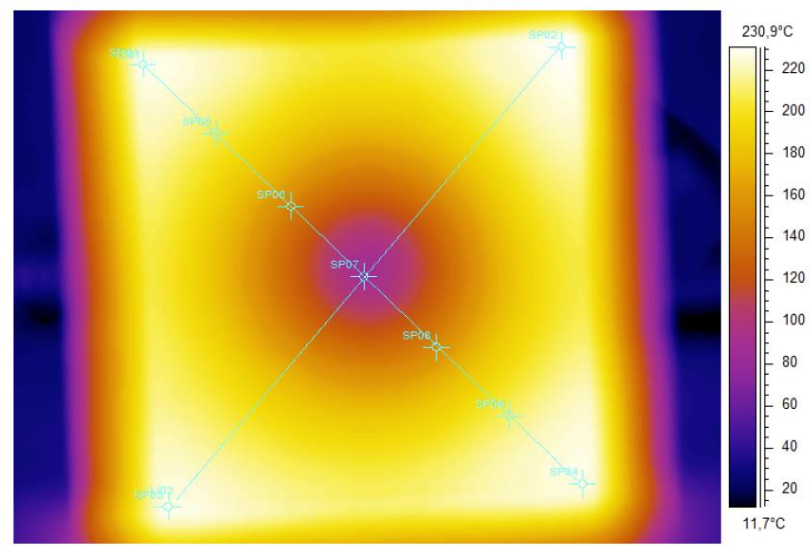

Fig. 7. Surface temperature of the point cooler after 10 seconds of cooling, water used as cooling medium.

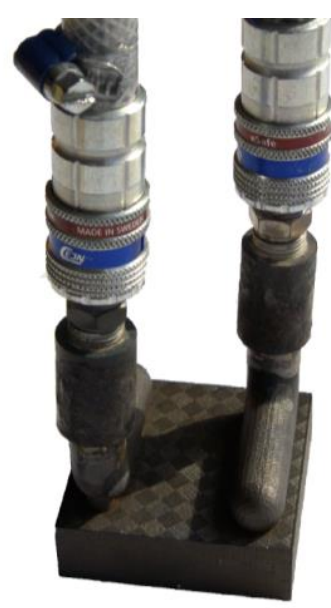

Fig. 6. The physical test objects for conformal cooling.

- the conformal cooler - is shown in Fig. 4. The smallest inner diameter of the tubes in the point cooler was $7.0 \mathrm{~mm}$, and the smallest inner diameter of the conformal cooler was $3.2 \mathrm{~mm}$. The physical test objects with connectors are shown in Fig. 5 and Fig. 6.

The coolers were tested with water and air as cooling medium. The water pressure at the inlet hose was 5 bar. For the point cooler the water flow rate was $13 \mathrm{l} / \mathrm{min}$ and for the conformal cooler the flow rate was $16 \mathrm{l} / \mathrm{min}$. Air cooling was carried out with a pressure of 7 bar.

The cooling effect tests were carried out by measuring the surface temperature during cooling from $300^{\circ} \mathrm{C}$ to room temperature. The objects were heated in a heat treatment oven for a sufficient time. Then, the hoses for the coolant were connected to the inlet and outlet channels and the valve for the coolant was opened.

During the cooling time the bottom of the prismatic object was photographed with a ThermaCam SC640 for measuring the surface temperature. The software ThermaCam Researcher Professional was used to analyze the results, and the temperature was measured at seven points along a diagonal line

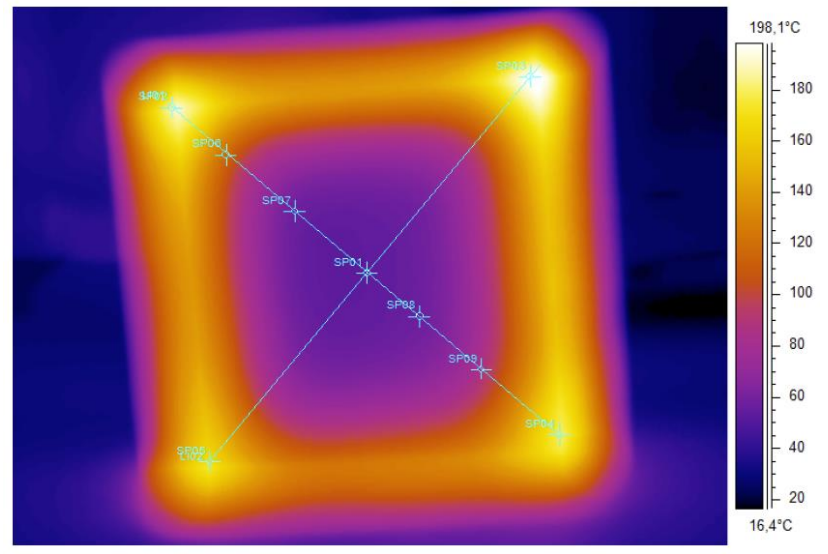

Fig. 8. Surface temperature of the conformal cooler after 10 seconds of cooling, water used as cooling medium. 

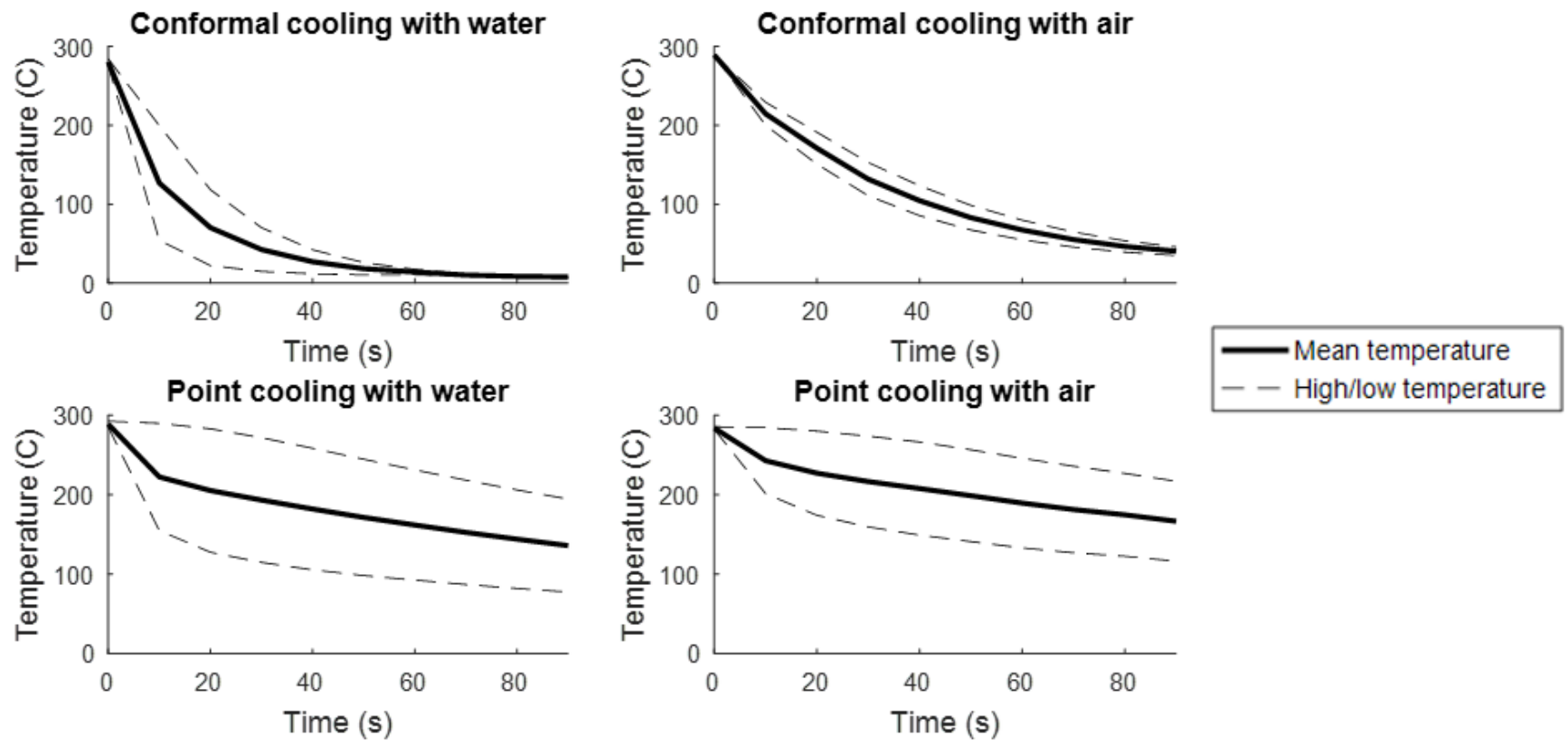

Fig. 9. Surface temperature of the conformal and point coolers during cooling experiments, water and air used as cooling medium. High temperature is measured in the corner of the test specimen, while the low temperature is measured in the center of the test specimen.

on the bottom of the prismatic part. Examples of the temperature measurements of the test objects with thermal imaging are shown in Fig. 7 and Fig. 8.

\section{B. Results and discussion}

A large difference is found between the two cooling systems. The measurements shown in Fig. 8 and 9 shows clearly that the temperature is more uniform for the conformal cooling than for the point cooling. The maximum temperature difference between warmest and coldest point during cooling was up to $180^{\circ} \mathrm{C}$ for the point cooler and up to $100^{\circ} \mathrm{C}$ for the conformal cooler when water cooling was used.

Fig. 9 shows the cooling curves for the point cooler and the conformal cooler. The curves show the mean temperature on the surface, and the highest and lowest temperatures. Cooling curves for both water cooling and air cooling are shown.

The conformal cooler gives a fast and uniform cooling. We see that even with the use of air as cooling medium the conformal cooler is more efficient than the conventional technique of using point cooler with water.

Compressed air is more expensive than water, but from a safety point of view, the use of air is a better choice in die casting due to the risk of an explosion if water comes in contact with the molten aluminium. The use of compressed air is safe and would therefore allow reduced wall thickness between cooling channels and mould cavity, giving larger flexibility in mould design.
The experiments presented in this paper quantifies the cooling effect of conformal cooling. It is shown that there is a large potential of productivity improvements by use of additive manufacturing in moulds and dies.

\section{ACKNOWLEDGMENT}

The authors thank Benteler Automotive Farsund AS and the Research Council of Norway for supporting this work through the NextForm project, Grant No. 235241.

\section{REFERENCES}

[1] M. Pereira, M. Williams, W. Du Preez, "Application of laser additive manufacturing to produce dies for aluminium high pressure die-casting", South African Journal of Industrial Engineering, vol. 23 (2), 2011.

[2] A. Armillotta, R. Baraggi, S. Fasoli, "SLM tooling for die casting with conformal cooling channels", International Journal of Advanced Manufacturing Technology, vol. 71 (1-4), pp. 573-83, 2014.

[3] D-G. Ahn, "Applications of laser assisted metal rapid tooling process to manufacture of moulding \& forming tools - state of the art", International Journal of Precision Engineering and Manufacturing, vol. 12 (5), pp. 925-938, 2011

[4] K. Boivie et al., "A practical case study for an intelligent tooling system", Rapid Product Development Association of South Africa (RAPDASA) conference, 2013.

[5] P. Fua et al., "Low-pressure die casting of magnesium alloy AM50: Response to process parameters", Journal of Materials Processing Technology, vol. 205 (1-3), pp. 224-234, 2008.

[6] V. Brøtan, O. Åsebø Berg, K. Sørby, "Additive manufacturing for enhanced performance of molds", 6th Conference on Learning Factories, 2016, Procidia CIRP, in press. 